\title{
Susceptibility to echinocandins of Candida spp. strains isolated in Italy assessed by European Committee for Antimicrobial Susceptibility Testing and Clinical Laboratory Standards Institute broth microdilution methods
}

\author{
Maria Teresa Montagna ${ }^{1 *}$, Grazia Lovero $^{1}$, Caterina Coretti ${ }^{1}$, Domenico Martinelli², Osvalda De Giglio ${ }^{1}$,
} Roberta latta ${ }^{3}$, Stella Balbino ${ }^{1}$, Antonio Rosato ${ }^{4}$ and Giuseppina Caggiano ${ }^{1}$

\begin{abstract}
Background: The echinocandins are recommended as first-line therapy for Candida species infections, but drug resistance, especially among Candida glabrata, is becoming more frequent. We investigated the antifungal susceptibility of anidulafungin, caspofungin, and micafungin against 584 isolates of Candida spp. (bloodstream, other sterile sites) collected from patients admitted to an Italian university hospital between 2000 and 2013. The susceptibility was evaluated using the broth microdilution method according to both the European Committee for Antimicrobial Susceptibility Testing (EUCAST EDef 7.2) and the Clinical Laboratory Standards Institute (CLSI M27-A3). The echinocandin susceptibilities were assessed on the basis of the species-specific clinical breakpoints proposed by the EUCAST version 6.1 and CLSI M27-S4 documents. The two methods were comparable by assessing essential agreement (EA), categorical agreement (CA), and Spearman's correlation analysis (rho, r).
\end{abstract}

Results: The modal minimum inhibitory concentrations (MICs; $\mu \mathrm{g} \cdot \mathrm{mL}^{-1}$ ) values by both methods (EUCAST/CLSI) for anidulafungin, caspofungin, and micafungin for each species were, respectively, as follows: C. albicans, 0.03/0.12, 0.016/0.5, and 0.016/0.008; C. parapsilosis complex, 2/1, 2/2, and 2/1; C. tropicalis, 0.06/0.12, 0.06/0.12, and 0.06/0.12; C. glabrata complex, 0.03/0.25, 0.06/0.12, and 0.03/0.06; C. guilliermondii, 2/1, 2/2, and 2/2; and C. krusei, 0.06/0.12, $0.12 / 0.5$, and 0.06/0.12. The overall resistance rates for EUCAST/CLSI were as follows: anidulafungin, 2.5/0.9\%; caspofungin, breakpoint not available/3.8 \%; micafungin, $2.7 / 1.5 \%$.

Candida glabrata complex was the least susceptible to all three echinocandins, and the percentages of resistant isolates by EUCAST/CLSI were as follows: anidulafungin, 13.5/2.7 \%; caspofungin, breakpoint not available/16.2 \%; micafungin, 18.9/13.5\%. The overall EA was $93 \%$ for micafungin, $92 \%$ for anidulafungin, and $90 \%$ for caspofungin. The CA was $>90 \%$ for all organism-drug combinations with the exception of C. glabrata and anidulafungin ( $89 \%$ ). Spearman's rho for EUCAST/CLSI was $0.89(p<0.001)$ for caspofungin, $0.85(p<0.001)$ for anidulafungin, and 0.83 for micafungin $(p<0.001)$.

Conclusions: Independent of the procedure applied, no alarming resistance to the tested agents was found, although a reduced susceptibility was detected for C. glabrata complex. The EUCAST and CLSI methods produce similar MICs, indicating that using one method or the other should not result in susceptibilities different enough to affect treatment decisions.

Keywords: Candida spp., EUCAST, CLSI, Anidulafungin, Caspofungin, Micafungin, Antifungal

\footnotetext{
*Correspondence: mariateresa.montagna@uniba.it

1 Department of Biomedical Science and Human Oncology, Hygiene Section, University of Bari "Aldo Moro", Piazza Giulio Cesare 11, Bari, Italy

Full list of author information is available at the end of the article
} 


\section{Background}

The echinocandins (anidulafungin [AND], caspofungin [CSP], and micafungin $[\mathrm{MCF}]$ ) are lipopeptides that inhibit glucan synthase, which is responsible for the biosynthesis of $\beta-1,3-D$-glucan, a major structural component of fungal cell walls. These drugs demonstrate fungicidal activity against most species of Candida and are effective against azole-resistant yeasts and Candida-forming biofilms [1-5]. The Infectious Diseases Society of America [6] and the European Society of Clinical Microbiology and Infectious Diseases [7] guidelines for the management of Candida infection recommend echinocandins for firstline therapy [8].

Two reference methods analyze the susceptibility of yeasts to echinocandins: the broth microdilution (BMD) method designed by the Clinical Laboratory Standards Institute (CLSI) $[9,10]$ and the method proposed by the European Committee for Antimicrobial Susceptibility Testing (EUCAST) [11-13]. These methods have some aspects in common: the use of BMD, the use of RPMI 1640 broth as a basal medium, a 24-h incubation period, and a prominent inhibition (50\% relative to the growth control) minimum inhibitory concentration (MIC) endpoint criterion. The main differences are as follows: inoculum density (CLSI, $0.5 \times 10^{3}$ to $2.5 \times 10^{3}$ cells $\cdot \mathrm{mL}^{-1}$; EUCAST, $0.5 \times 10^{5}$ to $2.5 \times 10^{5}$ cells $\cdot \mathrm{mL}^{-1}$ ); glucose content of the medium (CLSI, $0.2 \%$; EUCAST, $2.0 \%$ ); microdilution wells (CLSI, round-bottom wells; EUCAST, flat-bottom wells); and endpoint reading (CLSI, visual reading; EUCAST, spectrophotometric reading). Moreover, EUCAST breakpoints for AND and MCF are lower than the CLSI breakpoints; additionally, EUCAST has not proposed clinical breakpoints for CSP.

Until now, in Italy, there is not an extensive study concerning the in vitro susceptibility of echinocandins against Candida isolates using the EUCAST method [14]. The aims of this study are: i) to determine the susceptibilities of Candida spp. to AND, CSP, and MCF using both the EUCAST and CLSI methods; and ii) to compare the performance of both methods by assessing the essential agreement (EA) and categorical agreement (CA) levels.

\section{Results and discussion}

In each experiment, the MIC values of the quality control strains fell within the established ranges published for both methods $[9,13]$. The modal MIC $\left(\mu \mathrm{g} \cdot \mathrm{mL}^{-1}\right.$; 15 repetitions) values by EUCAST/CLSI for AND, CSP, and MCF for each control strain were, respectively, as follows: C. krusei ATCC 6258, 0.06/0.12, 0.5/0.5, and $0.12 / 0.12$; C. parapsilosis ATCC 22019, 1/1, 0.5/1, and $0.5 / 0.5$. Table 1 summarizes the in vitro susceptibility values of 584 clinical isolates of Candida species to echinocandins as determined by the CLSI and EUCAST
BMD methods, as well as EA, and CA between two methods for each echinocandin and Candida species. Generally, the MIC values for all three echinocandins were low and below the susceptibility breakpoint, regardless of the method used. As shown by other authors [15], the MIC values for AND (geometric mean MIC EUCAST/CLSI, $0.16 / 0.22 \mu \mathrm{g} \cdot \mathrm{mL}^{-1}$ ) and MCF (geometric mean MIC EUCAST/CLSI, 0.13/0.14 $\mu \mathrm{g} \cdot \mathrm{mL}^{-1}$ ) were lower those for CSP (geometric mean MIC EUCAST/CLSI, $0.29 / 0.33 \mu \mathrm{g} \cdot \mathrm{mL}^{-1}$ ) by both assay, suggesting that they have superior in vitro potency. When the species-specific clinical breakpoints were applied, the overall resistance rates for EUCAST/CLSI were as follows: AND, 2.5/0.9 \%; CSP, breakpoint not available/ $3.8 \%$; MCF, $2.7 / 1.5 \%$. These data are consistent with those reported previously $[14,16-18]$ and document the excellent potency and spectrum of echinocandins against most Candida spp. Of the 22 CSP resistant isolates by CLSI, five were MCF resistant, three were AND resistant, and one was resistant both AND and MCF; this discrepant susceptibility pattern is according to other studies [17, 19]. Given the mechanism of action that is shared among the echinocandins, it is biologically unlikely that such large percentages of isolates are non susceptible to CSP but remain susceptible to AND and MCF. A potential explanation for this finding may be the technical issues associated with the in vitro testing of CSP rather than a true difference in antifungal activity [20]. For this reason, neither EUCAST nor CLSI procedures recommend the use of CSP for antifungal susceptibility testing, while AND and MCF as markers for CSP susceptibility [11, 21].

The trend in the rate of resistance to echinocandins was analyzed: no significant trend was observed for each drug over the studied 14-year period, by both methods.

The MIC values were the highest for C. parapsilosis complex and C. guilliermondii by the two methods. A natural polymorphism occurring in the hot spot, one region of FKS1 has been suggested to be responsible for the reduced echinocandin susceptibilities of these species [22]. Unlike yeasts with acquired FKS mutations, these strains respond well to standard therapy presumably because the polymorphism only weakly affects the sensitivity of glucan synthase for drug [5]. Moreover, the good clinical response of $C$. parapsilosis may be due to its less virulent, reduced capacity to invade the deep tissue, and the high probability of therapeutic success if central venous catheter is removed [15].

In our study, the resistance to AND (EUCAST/CLSI, 13.5/2.7 \%), CSP (EUCAST/CLSI, breakpoint not available/16.2 \%), and MCF (EUCAST/CLSI, 18.9/13.5 \%) was most prominent among $C$. glabrata complex isolates. Similarly, the SENTRY Antimicrobial Surveillance Program reported echinocandin resistance of 8.0-9.3\% 
Table 1 Agreement between the results of the European Committee for Antimicrobial Susceptibility Testing and Clinical Laboratory Standards Institute broth microdilution methods for anidulafungin, caspofungin, and micafungin

\begin{tabular}{|c|c|c|c|c|c|c|c|c|c|c|c|}
\hline \multirow[t]{2}{*}{ Isolates (No.) } & \multirow{2}{*}{$\begin{array}{l}\text { Antifungal } \\
\text { drug }\end{array}$} & \multirow{2}{*}{$\begin{array}{l}\text { BMD } \\
\text { method }\end{array}$} & \multicolumn{4}{|l|}{$\mathrm{MIC}\left(\mu \mathrm{g} \mathrm{mL}^{-1}\right)$} & \multirow{2}{*}{$\begin{array}{l}\text { No. } \\
\text { (\%) S }\end{array}$} & \multirow{2}{*}{$\begin{array}{l}\text { No. } \\
(\%) \text { I }\end{array}$} & \multirow{2}{*}{$\begin{array}{l}\text { No. } \\
(\%) R\end{array}$} & \multirow{2}{*}{$\begin{array}{l}\text { EA } \\
(\%)\end{array}$} & \multirow{2}{*}{$\begin{array}{l}\text { CA } \\
(\%)\end{array}$} \\
\hline & & & Range & Mode & GM & $\mathrm{MIC}_{90}$ & & & & & \\
\hline \multirow[t]{6}{*}{ C. albicans (251) } & \multirow[t]{2}{*}{ Anidulafungin } & $\mathrm{CLSI}$ & $\leq 0.008-2$ & 0.12 & 0.03 & 0.12 & $246(98)$ & $2(0.8)$ & $3(1.2)$ & 90 & 99 \\
\hline & & EUCAST & $\leq 0.008-2$ & 0.03 & 0.02 & 0.03 & 247(98.4) & NA & $4(1.6)$ & & \\
\hline & \multirow[t]{2}{*}{ Micafungin } & CLSI & $\leq 0.008-1$ & 0.008 & 0.01 & 0.12 & $241(96)$ & $7(2.8)$ & $3(1.2)$ & 92 & 98 \\
\hline & & EUCAST & $\leq 0.008-0.5$ & 0.016 & 0.01 & 0.016 & 244(97.2) & NA & $7(2.8)$ & & \\
\hline & \multirow[t]{2}{*}{ Caspofungin } & CLSI & $\leq 0.008-4$ & 0.5 & 0.17 & 0.5 & 180(71.7) & $63(25.1)$ & $8(3.2)$ & 87 & NA \\
\hline & & EUCAST & $\leq 0.008-2$ & 0.016 & 0.13 & 0.5 & - & - & - & & \\
\hline \multirow{6}{*}{$\begin{array}{l}\text { C. parapsilosis } \\
\text { complex (224) }\end{array}$} & \multirow[t]{2}{*}{ Anidulafungin } & CLSI & $0.03-2$ & 1 & 1.14 & 2 & $224(100)$ & 0 & 0 & 96 & 100 \\
\hline & & EUCAST & $0.06-2$ & 2 & 1.78 & 2 & 0 & $224(100)$ & 0 & & \\
\hline & \multirow[t]{2}{*}{ Micafungin } & CLSI & $0.016-2$ & 1 & 0.96 & 2 & $224(100)$ & 0 & 0 & 95 & 100 \\
\hline & & EUCAST & $0.016-2$ & 2 & 1.62 & 2 & 0 & $224(100)$ & 0 & & \\
\hline & \multirow[t]{2}{*}{ Caspofungin } & CLSI & $0.25-8$ & 2 & 1.62 & 2 & 217(96.9) & $6(2.7)$ & $1(0.4)$ & 98 & NA \\
\hline & & EUCAST & $0.06-8$ & 2 & 1.82 & 2 & - & - & - & & \\
\hline \multirow[t]{6}{*}{ C. tropicalis (46) } & \multirow[t]{2}{*}{ Anidulafungin } & CLSI & $\leq 0.008-1$ & 0.12 & 0.09 & 0.25 & $45(97.8)$ & 0 & $1(2.2)$ & 91 & 93 \\
\hline & & EUCAST & $\leq 0.008-0.25$ & 0.06 & 0.05 & 0.06 & $42(91.3)$ & NA & $4(8.7)$ & & \\
\hline & \multirow[t]{2}{*}{ Micafungin } & CLSI & $\leq 0.008-1$ & 0.12 & 0.07 & 0.5 & $37(80.4)$ & $(17.4)$ & $1(2.2)$ & 91 & NA \\
\hline & & EUCAST & $0.016-2$ & 0.06 & 0.11 & 0.5 & - & - & - & & \\
\hline & \multirow[t]{2}{*}{ Caspofungin } & $\mathrm{CLSI}$ & $\leq 0.008-2$ & 0.12 & 0.12 & 1 & $34(74)$ & $6(13)$ & $6(13)$ & 89 & NA \\
\hline & & EUCAST & $\leq 0.008-2$ & 0.06 & 0.11 & 1 & - & - & - & & \\
\hline \multirow{6}{*}{$\begin{array}{l}\text { C. glabrata } \\
\text { complex (37) }\end{array}$} & \multirow[t]{2}{*}{ Anidulafungin } & CLSI & $\leq 0.008-4$ & 0.25 & 0.13 & 0.25 & $24(64.9)$ & $12(32.4)$ & $1(2.7)$ & 72 & 89 \\
\hline & & EUCAST & $\leq 0.008-2$ & 0.03 & 0.06 & 0.12 & $32(86.5)$ & NA & $5(13.5)$ & & \\
\hline & \multirow[t]{2}{*}{ Micafungin } & CLSI & $\leq 0.008-1$ & 0.06 & 0.06 & 0.12 & $28(75.7)$ & $4(10.8)$ & $5(13.5)$ & 83 & 95 \\
\hline & & EUCAST & $\leq 0.008-2$ & 0.03 & 0.05 & 0.12 & $30(81.1)$ & NA & $7(18.9)$ & & \\
\hline & Caspofungin & CLSI & $\leq 0.008-4$ & 0.12 & 0.18 & 1 & $21(56.8)$ & $10(27)$ & $6(16.2)$ & 80 & NA \\
\hline & & EUCAST & $\leq 0.008-2$ & 0.06 & 0.17 & 2 & - & - & - & & \\
\hline C. guilliermondii (15) & Anidulafungin & CLSI & $1-2$ & 1 & 1.45 & 2 & $14(93.3)$ & $1(6.7)$ & 0 & 100 & NA \\
\hline & & EUCAST & $1-2$ & 2 & 1.91 & 2 & - & - & - & & \\
\hline & Micafungin & CLSI & $0.5-2$ & 2 & 1.45 & 2 & $15(100)$ & 0 & 0 & 100 & NA \\
\hline & & EUCAST & $0.5-2$ & 2 & 1.52 & 2 & - & - & - & & \\
\hline & Caspofungin & CLSI & $0.12-4$ & 2 & 1.74 & 2 & $14(93.3)$ & $1(6.7)$ & 0 & 93 & NA \\
\hline & & EUCAST & $1-2$ & 2 & 1.74 & 2 & - & - & - & & \\
\hline C. krusei (11) & Anidulafungin & CLSI & $0.12-0.5$ & 0.12 & 0.14 & 0.12 & $10(90.9)$ & $1(9)$ & 0 & 90 & 91 \\
\hline & & EUCAST & $0.06-0.12$ & 0.06 & 0.06 & 0.06 & 10(90.9) & NA & $1(9.1)$ & & \\
\hline & Micafungin & CLSI & $0.06-0.5$ & 0.12 & 0.13 & 0.12 & $11(100)$ & 0 & 0 & 100 & NA \\
\hline & & EUCAST & $0.03-0.5$ & 0.06 & 0.08 & 0.25 & - & - & - & & \\
\hline & Caspofungin & CLSI & $0.12-2$ & 0.5 & 0.44 & 2 & $6(54.5)$ & $4(36.4)$ & $1(9.1)$ & 90 & NA \\
\hline & & EUCAST & $0.06-2$ & 0.12 & 0.23 & 1 & - & - & - & & \\
\hline
\end{tabular}

"-" denotes that no breakpoints have yet been established; BMD, broth microdilution; CLSI, Clinical and Laboratory Standards Institute; EUCAST, European Committee on Antimicrobial Susceptibility Testing; MIC, minimum inhibitory concentration; GM, geometric mean; EA, essential agreement; $C A$, categorial agreement; NA, Not applicable; S, susceptible; I, intermediate; R, resistant

among 1669 C. glabrata from blood stream infections [23]; moreover, in a 10-year survey at the Duke university hospital echinocandin resistance rate increased from 4.9 to $12.3 \%$ in 2001-2010 [24]. Prolonged therapy with these drugs has been suggested to be a potential cause for decreased susceptibility to echinocandins among isolates of C. glabrata [22-27].

A decrease in the activity of AND (EUCAST/CLSI, 8.7/2.2 \%), MCF (EUCAST/CLSI, breakpoint not available/2.2\%), and CSP (EUCAST/CLSI, breakpoint not 
available/13\%) was also observed among isolates of $C$. tropicalis.

For most isolates, the echinocandin MICs obtained by the EUCAST method tended to be one twofold dilution lower than those obtained by the CLSI method. The reason for this result could be related to known differences between the two methods: the higher carbohydrate content in the RPMI and higher inoculum could be responsible for the lowering of the EUCAST results [28].

The overall EA was very high: $93 \%$ for MCF, $92 \%$ for AND, and $90 \%$ for CSP. The Spearman's correlation analysis shows a significant positive correlation between EUCAST and CLSI MICs $(r=0.85, p<0.001$ for AND; $r$ $=0.89, p<0.001$ for CSP; $r=0.8, p<0.001$ for MCF). This finding was consistent with the results of previous global multicenter studies by Pfaller et al. $[29,30]$ and confirms the high level of EA between the reference procedures. The rates of EA were also high when results were analyzed per species, and the worst EA (72\%) was for C. glabrata complex tested against AND. A good CA was also observed for all organism-drug combinations, ranging from 89 to $100 \%$. The lowest CA was for $C$. glabrata complex isolates to AND, where five (13.5\%) isolates were resistant when applying EUCAST breakpoints as opposed to one $(2.7 \%)$ when applying the CLSI breakpoints. The meaning of this in vitro finding is not clear and needs to be clarified in more detail. The best CA was for C. parapsilosis complex to AND and MCF.

\section{Conclusions}

Our study had some limitations. First, none of our strains were characterized with respect to echinocandin resistance mechanisms. Second, the lack of EUCAST species-specific breakpoints for CSP precludes a more standard comparison for the assessment of the CA. Third, this study was an observational laboratory based survey, therefore no data was available as to the type and duration of antifungal therapy. Nevertheless, to the best of our knowledge, this is the first study on this topic to be carried out in Italy. Our data show that the EUCAST and CLSI echinocandin MIC values for Candida isolates from patients in Italy are in accordance with the worldwide epidemiology of Candida strains [14, 16-18]. Likewise, the echinocandins were active in vitro against the majority of Candida species tested in this study; a reduced susceptibility, as has been noted elsewhere [23, 24], was detected for C. glabrata complex and C. tropicalis. MIC values obtained by the CLSI and EUCAST methods are comparable for the testing of echinocandins against Candida species. Regression analysis confirms the close proximity of the MICs generated by each method. In most cases, the MIC differences between standard procedures are small enough that using one method or the other should not result in susceptibilities that are different enough to affect treatment decisions. The clinical implications regarding the improvement of the susceptibility tests to echinocandins are significant, since accurate data are important in defining differences in clinical efficacy among AND, CSP and MCF, thereby supporting the most appropriate choice of early antifungal treatment towards a better prognosis. Further efforts are needed to harmonize the two standard procedures.

\section{Methods}

\section{Clinical isolates}

Between January 2000 and December 2013, a total of 597 clinical isolates of Candida spp. (bloodstream and other sterile sites) were collected from patients admitted to a large Italian university hospital. Of these, the six most common Candida species were tested against echinocandins (251 C. albicans, 224 C. parapsilosis, 46 C. tropicalis, 37 C. glabrata, 15 C. guilliermondii, 11 C. krusei), for a total of 584 isolates recovered from the following wards: intensive care unit $(n=288)$, haematology $(n=99)$, internal medicine $(n=88)$, surgery $(n=85)$, and oncology $(n=24)$. For this study, we did not use any additional data or samples other than those obtained through routine laboratory collection. Therefore, neither ethical approval nor patient consent was considered necessary. Registered data were managed in accordance with the Italian data protection laws (privacy law). The isolates were identified using standard procedures (i.e., morphology on cornmeal agar plates, germ-tube production in serum, and ability to grow at $37{ }^{\circ} \mathrm{C}$ and $42{ }^{\circ} \mathrm{C}$ ) and biochemical analysis using the ID32C and VITEK-2 System (Biomérieux, Marcy l'Etoile, France). Each isolate represented a unique strain from a single patient and was frozen at $-80{ }^{\circ} \mathrm{C}$ until the analysis. Prior to being tested, each isolate was subcultured on Sabouraud dextrose agar plates (BioMèrieux) to ensure purity, viability, and optimal growth characteristics.

\section{Susceptibility testing}

AND (Pfizer Pharmaceuticals, Groton, CT, USA), CSP (Merck \& Co., Inc., Whitehouse Station, NJ, USA), and MCF (Astellas Pharma, Tokyo, Japan) were obtained as standard powders. A single lot of pure substance for each of the three echinocandins was used. Stock solutions were prepared in dimethyl sulfoxide (Sigma, St. Louis, MO, USA) for each echinocandin, taking into account the potencies of the powders.

CLSI BMD testing was performed according to document M27-A3 [9, 10] using RPMI 1640 medium with $0.2 \%$ glucose (Sigma) and 0.165 M MOPS, an inoculum of $0.5 \times 10^{3}$ to $2.5 \times 10^{3}$ cells $\cdot \mathrm{mL}^{-1}$, and incubation at $35{ }^{\circ} \mathrm{C}$. The MIC values were determined visually after $24 \mathrm{~h}$ of incubation as the lowest concentration of drug 
Table 2 CLSI (document M27-S4) and EUCAST (version 6.1) antifungal breakpoints for Candida species

\begin{tabular}{|c|c|c|c|}
\hline \multirow[b]{2}{*}{ Species } & \multicolumn{3}{|c|}{ MIC $(\mu \mathrm{g} / \mathrm{ml})$ breakpoint for susceptibility/resistance } \\
\hline & Anidulafungin & Caspofungin & Micafungin \\
\hline \multicolumn{4}{|c|}{ C. albicans } \\
\hline CLSI & $\leq 0.25 / \geq 1$ & $\leq 0.25 / \geq 1$ & $\leq 0.25 / \geq 1$ \\
\hline EUCAST & $\leq 0.03 />0.03$ & - & $\leq 0.016 />0.016$ \\
\hline \multicolumn{4}{|c|}{ C. parapsilosis } \\
\hline CLSI & $\leq 2 / \geq 8$ & $\leq 2 / \geq 8$ & $\leq 2 / \geq 8$ \\
\hline EUCAST & $\leq 0.002 />4$ & - & $\leq 0.002 />2$ \\
\hline \multicolumn{4}{|c|}{ C. glabrata } \\
\hline CLSI & $\leq 0.12 / \geq 0.5$ & $\leq 0.12 / \geq 0.5$ & $\leq 0.06 / \geq 0.25$ \\
\hline EUCAST & $\leq 0.06 />0.06$ & - & $\leq 0.03 />0.03$ \\
\hline \multicolumn{4}{|c|}{ C. tropicalis } \\
\hline CLSI & $\leq 0.25 / \geq 1$ & $\leq 0.25 / \geq 1$ & $\leq 0.25 / \geq 1$ \\
\hline EUCAST & $\leq 0.06 />0.06$ & - & - \\
\hline \multicolumn{4}{|c|}{ C. gulliermondii } \\
\hline CLSI & $\leq 2 / \geq 8$ & $\leq 2 / \geq 8$ & $\leq 2 / \geq 8$ \\
\hline EUCAST & - & - & - \\
\hline \multicolumn{4}{|l|}{ C. krusei } \\
\hline CLSI & $\leq 0.25 / \geq 1$ & $\leq 0.25 / \geq 1$ & $\leq 0.25 / \geq 1$ \\
\hline EUCAST & $\leq 0.06 />0.06$ & - & - \\
\hline
\end{tabular}

"-" denotes that no breakpoints have yet been established

that caused a significant diminution ( $\geq 50 \%$ inhibition) of growth below control levels.

EUCAST BMD testing was performed according to document EDef 7.2 [available on the EUCAST website: http://www.eucast.org] using RPMI 1640 medium supplemented with glucose to a final concentration of $2 \%$, an inoculum of $0.5 \times 10^{5}$ to $2.5 \times 10^{5}$ cells $\cdot \mathrm{mL}^{-1}$, and incubation at $35^{\circ} \mathrm{C}$. MIC values were determined with a spectrophotometer (wavelength of $450 \mathrm{~nm}$; ETI System Fast Reader ELX, Biotek, US) after $24 \mathrm{~h}$ of incubation as the lowest concentration of drug that resulted in $>50 \%$ inhibition of growth relative to that of the growth control.

C. krusei ATCC 6258 and C. parapsilosis ATCC 22019 were used as quality control strains in each run according to the CLSI M27-A3 document [9].

\section{Interpretation and analysis of results}

The echinocandin susceptibilities were defined according to the species-specific clinical breakpoints proposed by the EUCAST version 6.1 [31] and CLSI M27-S4 documents [10] (Table 2). Differences in the temporal susceptibility rates were analysed by chi-square test for trend. MIC discrepancies of no more than \pm 2 -fold dilutions were used to calculate the EA. The CA was defined as the percentage of the discrepancy in the number of resistant isolates based on the existence of interpretative breakpoints [16]. Moreover, to determine the correlation between the methods, Spearman's rank correlation coefficient (rho, r), and its corresponding $\mathrm{p}$ value was performed by plotting EUCAST versus CLSI MICs. The level of significance was set at a $\mathrm{p}$ value less than 0.05 . Statistical analysis of data was carried out using STATA MP 11.2 for Mac Os X (SPSS Inc., Chicago, IL, USA).

\section{Abbreviations}

BMD: Broth microdilution; CLSI: Clinical Laboratory Standards Institute; EUCAST: European Committee for Antimicrobial Susceptibility Testing; AND: Anidulafungin; CSP: Caspofungin; MCF: Micafungin; EA: Essential agreement; CA: Categorical agreement; MIC: Minimum inhibitory concentration.

\section{Competing interests}

The authors declare that they have no competing interests.

\section{Authors' contributions}

MTM and CG designed the study; MTM, GL and GC analyzed data and wrote the manuscript; CC, ODG contributed to collect clinical samples and revised manuscript; SB, Rl, AR carried out the susceptibility testing. DM and GL performed statistical analysis. All authors read and approved the final manuscript.

\section{Authors' information}

MTM: Full professor; GL: PhD; CC: Graduate student; BS: Graduate student; DM: Assistant professor; ODG: PhD; Rl: PhD; AR: Assistant professor; GC: Assistant professor.

\section{Acknowledgements}

This study was supported by an unrestricted educational grant from Pfizer Italia.

\section{Author details}

${ }^{1}$ Department of Biomedical Science and Human Oncology, Hygiene Section, University of Bari "Aldo Moro", Piazza Giulio Cesare 11, Bari, Italy.

${ }^{2}$ Department of Medical and Surgical Sciences, Hygiene Section, University of Foggia, Via Gramsci 89-91, 71100 Foggia, Italy. ${ }^{3}$ Department of Veterinary Medicine, University of Bari Aldo Moro, Str. Prov. per Casamassima Km 3, Valenzano, Bari, Italy. ${ }^{4}$ Department of Pharmacy-Drug Sciences, University of Bari Aldo Moro, Campus-Via Orabona, 4, Bari, Italy.

Received: 9 January 2015 Accepted: 11 May 2015

Published online: 20 May 2015

\section{References}

1. Perlin DS. Current perspectives on echinocandin class drugs. Future Microbiol. 2011;6:441-57.

2. Pfaller MA, Diekema DJ, Andes D, Arendrup MC, Brown SD, Lockhart SR, et al. Clinical breakpoints for the echinocandins and Candida revisited: integration of molecular, clinical, and microbiological data to arrive at species-specific interpretive criteria. Drug Resist Updat. 2011:14:164-76

3. Spreghini E, Orlando F, Sanguinetti M, Posteraro B, Giannini D, Manso E, et al. Comparative effects of micafungin, caspofungin, and anidulafungin against a difficult-to-treat fungal opportunistic pathogen, Candida glabrata. Antimicrob Agents Chemother. 2012;56:1215-22.

4. Spreghini E, Orlando F, Tavanti A, Senesi S, Giannini D, Manso E, et al. In vitro and in vivo effects of echinocandins against Candida parapsilosis sensu stricto, Candida orthopsilosis and Candida metapsilosis. J Antimicrob Chemother. 2012;67:2195-202.

5. Perlin DS. Echinocandin resistance, susceptibility testing and prophylaxis: implications for patient management. Drugs. 2014;74:1573-85.

6. Pappas PG, Kauffman CA, Andes D, Benjamin Jr DK, Calandra TF, Edwards Jr JE, et al. Clinical practice guidelines for the managementof candidiasis: 2009 update by the Infectious Diseases Society of America. Clin Infect Dis. 2009;48:503-35. 
7. Ullmann AJ, Akova M, Herbrecht R, Viscoli C, Arendrup MC, Arikan-Akdagli S, et al. ESCMID* guideline for the diagnosis and management of Candida diseases 2012: adults with haematological malignancies and after haematopoietic stemcell transplantation (HCT). Clin Microbiol Infect. 2012;18 Suppl 7:53-67.

8. Leroux S, Ullmann AJ. Management and diagnostic guidelines for fungal diseases in infectious diseases and clinical microbiology: critical appraisal. Clin Microbiol Infect. 2013;19:1115-21.

9. Clinical and Laboratory Standards Institute. Reference method for broth dilution antifungal susceptibility testing of yeasts; third edition, M27-A3. Wayne, PA, USA: CLSI; 2008.

10. Clinical and Laboratory Standards Institute. Reference method for broth dilution antifungal susceptibility testing of yeasts; fourth informational supplement, M27-S4. Wayne, PA, USA: CLSI; 2012.

11. Subcommittee on Antifungal Susceptibility Testing (AFST) of the ESCMID European Committee for Antimicrobial Susceptibility Testing (EUCAST). EUCAST definitive document EDef 7.1: method for the determination of broth dilution MICs of antifungal agents for fermentative yeasts. Clin Microbiol Infect. 2008;14:398-405.

12. Arendrup MC, Cuenca-Estrella M, Lass-Florl C, Hope WW. EUCAST-AFST. EUCAST technical note on the EUCAST definitive document EDef 7.2: method for the determination of broth dilution minimum inhibitory concentrations of antifungal agents for yeasts EDef 7.2 (EUCAST-AFST). Clin Microbiol Infect. 2012;18:E246-7.

13. Arendrup MC, Cuenca-Estrella M, Lass-Flörl C, Hope WW, European Committee on Antimicrobial Susceptibility Testing-Subcommittee on Antifungal Susceptibility Testing (EUCAST-AFST). EUCAST technical note on Candida and micafungin, anidulafungin and fluconazole. Mycoses. 2014;57:377-9.

14. Tortorano AM, Prigitano A, Lazzarini C, Passera M, Deiana ML, Cavinato S, et al. A 1-year prospective survey of candidemia in Italy and changing epidemiology over one decade. Infection. 2013;41:655-62.

15. Beyda ND, Lewis RE, Garey KW. Echinocandin resistance in Candida species: mechanisms of reduced susceptibility and therapeutic approaches. Ann Pharmacother. 2012;46:1086-96.

16. Faria-Ramos I, Neves-Maia J, Ricardo E, Santos-Antunes J, Silva AT, Costade-Oliveira $\mathrm{S}$, et al. Species distribution and in vitro antifungal susceptibility profiles of yeast isolates from invasive infections during a Portuguese multicenter survey. Eur J Clin Microbiol Infect Dis. 2014;33:2241-7.

17. Guinea J, Zaragoza O, Escribano P, Martín-Mazuelos E, Pemán J, Sánchez-Reus F, et al. Molecular identification and antifungal susceptibility of yeast isolates causing fungemia collected in a population-based study in Spain in 2010 and 2011. Antimicrob Agents Chemother. 2014;58:1529-37.

18. Matsumoto E, Boyken L, Tendolkar S, McDanel J, Castanheira M, Pfaller M, et al. Candidemia surveillance in lowa: emergence of echinocandin resistance. Diagn Microbiol Infect Dis. 2014;79:205-8.

19. Pham CD, labal N, Bolden CB, Kuykendall RJ, Harrison LH, Farley MM, et al. Role of FKS Mutations in Candida glabrata: MIC values, echinocandin resistance, and multidrug resistance. Antimicrob Agents Chemother. 2014;58:4690-6.

20. Espinel-Ingroff A, Arendrup MC, Pfaller MA, Bonfietti LX, Bustamante B, Canton E, et al. Interlabo-ratory variability of caspofungin MICs for Candida spp. using CLSI and EUCAST methods: should the clinical laboratory be testing this agent? Antimicrob Agents Chemother. 2013;57:5836-42.

21. Arendrup MC, Perlin DS. Echinocandin resistance: an emerging clinical problem? Curr Opin Infect Dis. 2014;27:484-92.

22. Garcia-Effron G, Katiyar SK, Park S, Edlind TD, Perlin DS. A naturally occurring proline-to-alanine amino acid change in Fks1p in Candida parapsilosis, Candida orthopsilosis, and Candida metapsilosis accounts for reduced echinocandin susceptibility. Antimicrob Agents Chemother. 2008;52:2305-12.

23. Pfaller MA, Castanheira M, Lockhart SR, Ahlquist AM, Messer SA, Jones RN. Frequency of decreased susceptibility and resistance to echinocandins among fluconazole-resistant bloodstream isolates of Candida glabrata: results from the SENTRY Antimicrobial Surveillance Program (2006-2010) and the Centers for Disease Control and Prevention Population-Based Surveillance (2008-2010). J Clin Microbiol. 2012;50:1199-203.

24. Alexander BD, Johnson MD, Pfeiffer CD, Jiménez-Ortigosa C, Catania J, Booker $\mathrm{R}$, et al. Increasing echinocandin resistance in Candida glabrata: clinical failure correlates with presence of FKS mutations and elevated minimum inhibitory concentrations. Clin Infect Dis. 2013;56:1724-32.

25. Thompson GR, Wiederhold NP, Vallor AC, Villareal NC, Lewis JS, Patterson TF. Development of caspofungin resistance following prolonged therapy for invasive candidiasis secondary to Candida glabrata infection. Antimicrob Agents Chemother. 2008;52:3783-5.

26. Pfeiffer CD, Garcia-Effron G, Zaas AK, Perfect JR, Perlin DS, Alexander BD. Breakthrough invasive candidiasis in patients on micafungin. J Clin Microbiol. 2010;48:2373-80.

27. Cleary JD, Garcia-Effron G, Chapman SW, Perlin DS. Reduced Candida glabrata susceptibility secondary to an FKS1 mutation developed during candidemia treatment. Antimicrob Agents Chemother. 2008;52:2263-5.

28. Cuenca-Estrella M, Díaz-Guerra TM, Mellado E, Rodríguez-Tudela JL. Influence of glucose supplementation and inoculum size on growth kinetics and antifungal susceptibility testing of Candida spp. J Clin Microbiol. 2001;39:525-32.

29. Pfaller MA, Castanheira M, Diekema DJ, Messer SA, Moet GJ, Jones RN. Comparison of European Committee on Antimicrobial Susceptibility Testing (EUCAST) and Etest methods with the CLSI broth microdilution method for echinocandin susceptibility testing of Candida species. J Clin Microbiol. 2010:48:1592-9.

30. Pfaller MA, Castanheira M, Messer SA, Rhomberg PR, Jones RN. Comparison of EUCAST and CLSI broth microdilution methods for the susceptibility testing of 10 systemically active antifungal agents when tested against Candida spp. Diagn Microbiol Infect Dis. 2014;79:198-204.

31. Arendrup MC, Cuenca-Estrella M, Lass-Flörl C, Hope WW. Breakpoints for antifungal agents: an update from EUCAST focussing on echinocandins against Candida spp. and triazoles against Aspergillus spp. Drug Resist Updat. 2013;16:81-95.

\section{Submit your next manuscript to BioMed Central and take full advantage of:}

- Convenient online submission

- Thorough peer review

- No space constraints or color figure charges

- Immediate publication on acceptance

- Inclusion in PubMed, CAS, Scopus and Google Scholar

- Research which is freely available for redistribution 